Etat des lieux sur la gestion des déchets biomédicaux solides dans les formations sanitaires au Cameroun (Nord, Adamaoua, Est et Nord-Ouest) : Impacts Environnementaux et Sociaux.

\author{
Severin MBOG MBOG ${ }^{1,2 *}$ Olivier T SOSSO MAYI ${ }^{3}$, Dieudonne BITONDO ${ }^{1,4}$, Innocent NDOH \\ MBUE $^{1,4^{*}}$ \\ ${ }^{1}$ Department of Industrial Quality, Hygiene, Safety and Environment, National Advanced School for \\ Engineering, University of Douala, P.O Box 2701, Douala-Cameroon severinmbog.sm@gmail.com \\ ${ }^{2}$ Department of Plant Biology, Faculty of Sciences, University of Yaoundé I, P.O Box 331 Yaounde- \\ Cameroun \\ ${ }^{3}$ Laboratory of Thermal and Environment, Advanced Teacher's Training College for Technical Education, \\ University of Douala, P.O Box 1872 Douala-Cameroon \\ ${ }^{4}$ Laboratory of Energy, Materials, Modeling and Methods, National Advanced school for Engineering, \\ University of Douala, P.O Box 2701, Douala-Cameroon.
}

\title{
Résumé
}

Les déchets biomédicaux (DBM) constituent un problème sanitaire et environnemental. Aussi, une étude a été menée pour élaborer un système de gestion des déchets biomédicaux dont la mise en œuvre permettra d'améliorer la santé publique, tout en respectant les préoccupations environnementales, techniques et socioculturelles. Il s'agit d'une étude transversale descriptive qui s'est déroulée du 16 au 31 Janvier 2018. Un questionnaire soumis aux responsables des formations sanitaires (FOSA), chefs de services, surveillants de services et responsables de l'hygiène hospitalière et un entretien avec les personnels de soins et les opérateurs d'incinérateurs ont permis d'apprécier les mécanismes et les connaissances sur la gestion des DBM. Sur les 150 questionnaires distribués, 98 réponses ont été obtenues, soit un taux de réponse de 65,3\%. Un entretien avec 75 travailleurs directement impliqués dans la gestion des DBM et des observations sur la gestion des DBM au niveau des 86 services ont été réalisés. Les déchets tranchants et piquants et ceux de sang et de fluides étaient trouvés dans tous les services, à l'exception des pharmacies, les déchets pharmaceutiques dans 66 services, les déchets infectieux dans 49 services et les déchets anatomiques dans 11 services. Le tri des DBM était inadapté dans 83,5\% des FOSA et l'utilisation du système de codage par couleur inexistant dans les FOSA PBF et non PBF. Des boîtes de sécurité pour le recueil des objets tranchants et piquants étaient disponibles dans $62,5 \%$ des FOSA PBF et leur utilisation effective dans 31,1 \% d'entre eux. Ceux Dans la majorité des FOSA non PBF, un conditionnement inadapté était noté sous la forme d'une utilisation de bouteilles et poubelles en plastique pour le recueil et d'un remplissage des boîtes de sécurité avec des débordements. L'élimination des

Received: 14/02/2020

Accepted: 19/07/2020

DOI: https//dx.doi.org/10.4314/jcas.v16i1.2

(C) The Authors. This work is licensed under the Creative Commons Attribution 4.0 International Licence. 
DBM était effectuée dans de vieux incinérateurs ou des fours artisanaux, avec d’importantes émanations de fumées dans $80 \%$ des FOSA. Les conditions de travail étaient jugées mauvaises par 81,3\% des travailleurs interrogés et les équipements de protection individuelle disponibles dans seulement 25,3\% des FOSA non PBF et à 65\% dans les PBF. Les connaissances sur la gestion des DBM étaient jugées insuffisantes par 92,6\% des personnes interrogées et les risques sanitaires liés aux DBM connus par 90 $\%$. Toutefois les impacts sur le plan sanitaire et environnemental a été plus relevé dans les FOSA non PBF à $70 \%$ que chez les FOSA PBF à $30 \%$. A exception de l'Hôpital Régional de Bertoua qui faisant partie des FOSA PBF a présenté des impacts majeurs sur la santé et l'environnement.

Mots clés Déchets biomédicaux, Gestion, Pollution, Risques, Environnement•

\begin{abstract}
Biomedical waste (DBM) is a health and environmental problem. Also, a study was conducted to develop a biomedical waste management system whose implementation will improve public health, while respecting environmental concerns, technical and socio-cultural. This is a descriptive cross-sectional study that was conducted from the 16 th to $31^{\text {st }}$ January, 2018 A questionnaire sent to managers of health facilities (FOSA), department heads, supervisors and services responsible for hospital hygiene and interview with the personal care and incinerator operators were enjoying the mechanisms and knowledge management DBM. Of the 150 questionnaires sent, 98 responses were received, representing a $65.3 \%$ response rate. An interview with 75 workers directly involved in the management of DBM and observations on the management of DBM in the 86 services was performed. Sharp waste, blood and fluids were found in all services, except for pharmacies, pharmaceutical waste in 66 services, infectious waste in 49 services and anatomical waste in 11 services. Sorting DBM was inadequate in $83.5 \%$ of health facilities and the use of a non-existent color coding system in health facilities and non PBF PBF. safety boxes for the collection of sharps were available in $62.5 \%$ of FOSA PBF and their effective use in $31.1 \%$ of them. Those in the majority of non FOSA PBF, improper packaging was noted as use of bottles and plastic bins for collection and filling safety boxes with overflows. The elimination of DBM was done in old incinerators or artisanal ovens, with significant smoke leakage in $80 \%$ of health facilities. Working conditions were deemed bad by $81.3 \%$ of surveyed workers and personal protective equipment available in only $25.3 \%$ of non FOSA PBF and $65 \%$ in the PBF. Knowledge of the management of DBM were deemed insufficient by $92.6 \%$ of respondents and the health risks associated with DBM known by $90 \%$. However the impact on the health and environmental plan was more pointed in FOSA non PBF to $70 \%$ than in FOSA PBF to $30 \%$. A except Bertoua Regional Hospital that part of FOSA $\mathrm{PBF}$ presented major impacts on health and the environment.
\end{abstract}

Keywords Biomedical Waste, Management Pollution, Risks, Environment 
Introduction

Les pollutions imputables aux déchets biomédicaux (DBM) est sans doute l'une des plus complexes à résoudre. Ces déchets entraînent des sujétions particulières quant à leurs modalités de gestion. Une telle gestion, nécessite une politique d'hygiène appropriée, des moyens humains, matériels et financiers suffisants, du personnel formé et une réglementation adéquate pour pouvoir prévenir, si non réduire, tout risque pour les patients, les professionnels et l'écosystème [1].

Dans toutes formations sanitaires, une mauvaise gestion des déchets peut avoir des conséquences sur le personnel de santé, celui en charge de la gestion des déchets, l'environnement immédiat des centres de santé [7]. Au Cameroun comme dans la plupart des pays africains, la manipulation inappropriée des matériels infectés provenant des centres de santé, et la gestion inappropriée des déchets biomédicaux (collecte, stockage, traitement et élimination) constituent un risque non seulement pour le personnel hospitalier, les populations riveraines des structures sanitaires, mais aussi les services municipaux parfois qui reçoivent les déchets solides provenant de ces milieux de soins[19].

L'enquête menée en 2002 par l'OMS dans 22 pays en développement a révélé que la proportion d'établissements de soins n'appliquant pas les méthodes appropriées d'élimination des déchets de soins variait entre 18 et $64 \%$. Devant cette situation préoccupante, l'OMS a lancé en 2005 le défi mondial pour la sécurité des soins et proposé, comme principale stratégie, la gestion des DBM pour prévenir les différents risques engendrés [1].

C'est ainsi que le projet d'Appui aux Investissements dans le Secteur de la Santé (PAISS) a était mis sur pied pour avoir comme objectif premier de contribuer à l'amélioration de l'état général de la santé des populations à travers une plus grande performance des services de santé, améliorer la qualité de la santé maternelle et infantile, et réduire l'impact des maladies transmissibles

Les déchets biomédicaux (DBM), ou déchets de soins médicaux (DSM), comprennent tous les déchets issus des activités de diagnostic, de suivi, de traitements préventifs, curatifs et palliatifs dans le domaine de la médecine humaine et vétérinaire. Ils sont produits par les établissements de santé humaine, d'hygiène vétérinaire, de recherche et d'enseignement médical, les laboratoires d'essai ou de recherche clinique et les établissements de production ou d'essai de vaccin.

Les DBM sont classés en cinq catégories [17] :

- catégorie A : les DBM sans risques (ordures de bureaux, emballages, restes alimentaires);

- catégorie B : les DBM nécessitant une attention particulière (déchets anatomiques, déchets tranchants et piquants, déchets pharmaceutiques, déchets sanguins et fluides);

- catégorie $C:$ les déchets infectieux et hautement infectieux (déchets de laboratoires et cultures microbiologiques) ;

- catégorie D : les autres déchets dangereux (substances chimiques, gazeuses, liquides ou solides à haute teneur en métaux lourds) ;

- catégorie E : les DBM radioactifs (cobalt, technétium, iridium).

Les risques liés aux DBM sont d'ordre psychosocial, traumatique, infectieux, toxique, radioactif et environnemental. La gestion des DBM est décrite comme le processus visant à garantir l'hygiène des établissements de soins, la sécurité du personnel de santé et de la communauté. Elle inclut la planification, l'approvisionnement, la formation et le comportement du personnel de santé, l'utilisation correcte des outils, du matériel et des produits pharmaceutiques, les méthodes de traitement 
adaptées à l'intérieur ou à l'extérieur des établissements de soins et l'évaluation [9].

L'étude menée en 2008 par le MINSANTE/DPS au niveau des formations sanitaires a montré une gestion défectueuse des DBM et une absence totale de stratégies correctrices [12].

C'est dans ce cadre qu'il nous a paru nécessaire d'effectuer une étude sur la gestion des DBM au sein des formations sanitaires PBF et non PBF dans certaines régions du Cameroun dans la perspective d'y réduire les risques qui leur sont liés et d'améliorer les conditions d'hygiène et de sécurité dans l'environnement de travail. L'évaluation de l'amélioration de l'impact des maladies dans les centres de santé passe par une meilleure gestion des déchets biomédicaux.

\section{Matériel et méthodologie}

L'Etat des lieux de la gestion des DBM dans les formations sanitaires (FOSA) s'est essentiellement basée sur l'exploitation des divers documents de formulation et de mise en œuvre du projet d'une part, et sur la consultation des diverses parties prenantes du projet et la visite des FOSA d'autre part dans les régions NordOuest, de l'Est, de l'Adamaoua et du Nord.

L'étude s'est déroulée en quatre grandes phases ainsi qu'il suit :

- l'organisation de l'étude avec les responsables de l'Unité de coordination du PAISS ;

- la collecte et l'analyse des divers documents relatifs aux aspects environnementaux du projet : documents cadres (PAD, COPIL), rapports d'activités annuelles, rapports d'audit externe é, aide-mémoires des missions d'appui ;

-la consultations des divers parties prenantes dans Nord-Ouest, de l'Est, de l'Adamaoua et du Nord : responsables de l'UCP, Agences d'Achat de Performance (AAP), Fonds Régionaux pour la Promotion de la Santé (FRPS), Points Focaux PBF du projet (PF), Commune, Délégué du District de Santé (DDS) ;
- la visite de quelques FOSA dans les régions susmentionnées, afin d'identifier le niveau de la mise en œuvre effective du PBF sur la gestion des déchets biomédicaux (DB), d'évaluer les impacts des déchets biomédicaux sur l'environnement, proposer des mesures et actions concrètes à mener pour la gestion de ces déchets dangereux.

Un guide d'entretien et une grille d'observation présentés ont été élaborés pour les échanges avec les diverses parties prenantes et la collecte des données dans les FOSA. À la suite de chaque entretien a été élaboré un compte rendu des échanges. Une lettre a été adressée au préalable aux responsables des structures hospitalières pour obtenir une autorisation et un appui dans la réalisation de l'enquête.

La population de l'étude comprenait les responsables des hôpitaux (directeur, responsable des ressources humaines), les chefs des services médicaux, chirurgicaux, pharmaceutiques et des laboratoires, les surveillants de service, les responsables de l'hygiène hospitalière, les personnels de soins, les techniciens de surface et les opérateurs d'incinérateurs. Les outils de collecte des données étaient :

- un questionnaire adressé aux responsables des structures, chefs des services, surveillants des services et responsables de l'hygiène hospitalière pour apprécier les mécanismes de gestion des DBM ;

- un guide d'entretien à l'intention des opérateurs de DBM (techniciens de surface, opérateurs d'incinérateurs) et des personnels de soins (médecins, infirmiers, sages-femmes) pour apprécier leurs connaissances sur la gestion des DBM ;

- une grille d'observation pour mettre en relation et objectiver les informations recueillies lors des questionnaires, entretiens et observations au sein des services ; 
- un appareil photo numérique pour appuyer les observations au sein des services;

- une fiche de saisie des données pour leur traitement.

La méthode de recueil des données était basée sur les observations directes, le questionnaire, les entretiens et les photographies.

Les variables étudiées étaient les différentes catégories de DBM produites, les différentes étapes de gestion des DBM (tri, conditionnement, stockage, transport, élimination), les moyens de protection mis à la disposition du personnel, les connaissances et pratiques du personnel sur la gestion et les risques sanitaires liés aux DBM.

Les difficultés rencontrées étaient essentiellement liées au refus de certaines personnes à participer à l'enquête. Les données collectées ont été saisies à l'aide d'un logiciel Excel 2013.

\section{Résultats}

Les FOSA concernés par l'étude comprennent dans la Région du Nord-Ouest, quatre districts d'arrondissement où toutes les formations sanitaires présentes sont sous PBF. Il s'agit de Fundong, Ndop, Kumbo-est et Nkambe. Dans la ville de Bamenda spécifiquement aucune FOSA n'est sous PBF.Dans la région de l'Est, 13 FOSA PBF et 09 FOSA non PBF ont été visitées dans les localités de Bertoua, Dimako, Doumé, Diang, Belabo, Abong-Mbang.Dans les régions du nord et de l'Adamaoua, toutes les FOSA rencontrées sont non PBF. On dénombre 05 FOSA non PBF dans l'Adamaoua et 07 FOSA non PBF à Garoua.

Ces formations sanitaires reparties en 06 catégories : Hôpital Général et assimilé (catégorie 1), Hôpital central (catégorie 2), Hôpital Régional (catégorie 3), Hôpital de District (catégorie 4), Centre Médical d'Arrondissement (catégorie 5) Centres de Santé Intégrés/Centres de Santé Ambulatoire (catégorie 6). Le Département de la santé compte 2986 agents toutes catégories confondues.
Ces formations sanitaires PBF et non PBF de notre étude employaient 3833 travailleurs. Les administratifs représentaient $6,2 \%$ de ce personnel, les médecins $20,7 \%$, les pharmaciens $1,9 \%$, les infirmiers $43,6 \%$, les sages-femmes $2,7 \%$, les aides-infirmiers $13,5 \%$, les agents d'hygiène $0,5 \%$, les techniciens de surface 10,5 $\%$ et les opérateurs d'incinérateurs $0,4 \%$ (Tableau 2). Le responsable d'hygiène hospitalière dont $18,3 \%$ au niveau des FOSA de l'Est, 18,9 \% à Ngaoundéré, 19,6 \% à Garoua et 29,3\% au NordOuest.

Sur les 150 questionnaires administrés aux responsables des hôpitaux, chefs de service, surveillants de service et responsables de l'hygiène hospitalière, 130 réponses ont été obtenues, soit un taux de réponses de 75,3\%. Un entretien a été réalisé auprès de 75 travailleurs directement impliqués dans la gestion des DBM dans les FOSA PBF et non PBF dans les quatre Régions de l'étude, à savoir les personnels de soins, les techniciens de surface et les opérateurs d'incinérateurs (Tableau 3).

Des observations sur la gestion des DBM ont été également réalisées dans l'ensemble des services. Les déchets tranchants et piquants et ceux de sang et de fluides étaient retrouvés dans tous les services à l'exception des pharmacies, les déchets pharmaceutiques dans 66 services, les déchets infectieux dans 49 services et les déchets anatomiques dans 11 services.

Les déchets assimilables aux ordures ménagères (DAOM) étaient des cartons, papiers, sacs et pochettes en plastique, bouteilles d'eau minérale vides et restes alimentaires.

Les déchets infectieux comprenaient des lames de cultures microbiologiques, des tubes de culture et des milieux de culture. Les déchets anatomiques retrouvés étaient des cordons ombilicaux, placentas, fotus, pièces anatomiques et dents. 
Les déchets pharmaceutiques comprenaient des flacons vides de solutés, d'antibiotiques et de divers médicaments.

Les autres types de déchets produits étaient des films radiologiques, des solutions de fixation et de développement des films de radiologie, des colorants de laboratoires, des amalgames et des thermomètres médicaux. Les déchets les plus fréquemment retrouvés étaient des déchets tranchants et piquants, suivis de déchets de sang et de fluides, des déchets pharmaceutiques, des déchets infectieux et des déchets anatomiques.

Le tri était systématique dans 46,5\% de l'ensemble des FOSA PBF et non PBF, soit dans $19,8 \%$ à l'Est, dans 11,6\% au Nord-Ouest, dans $7 \%$ à l'Adamoua, dans 4,6\% au Nord (Tableau 1).

Le tri des DBM était inadapté (absence de séparation des DBM et des DAOM) dans 53,5\% de certains FOSA Non PBF à instart du District d'Abong Mbang, SCI d'ATOK, SCI d'Oboul I, SCI de Doumé et District de Diang. Ce tri inadapté concernait 16,3\% SCI, respectivement 12,8 \% de ceux CMA, 10,5 \% de ceux de l'hôpital Régional et 1,1\% de ceux de l'Hôpital Central.
Le système de codage par couleur des différentes catégories de déchets était inexistant dans toutes les formations sanitaires. Cependant, ce système de codage n'était pas utilisé au niveau d'autoclavables alors que les DASRI liquides étaient dilués dans de l'eau de Javel

Des boîtes de sécurité pour le recueil des objets tranchants et piquants étaient disponibles dans 82,5\% des FOSA PBF à savoir CMA de Dimako, Hôpital de district de doumé, Hôpital de district d'Abong Mbang, Hôpital de Reference de Bertoua et autres dans la zone de l'Est 32,5\%. De même dans la région du Nord-Ouest à $50 \%$ travers les FOSA telque: St Martin de Porres à Njinikom, Fundong Urban CSI, Njinikom CMA, Fundong District Hospital, Bangolan Baptist CSI.

Cependant, l'utilisation de ces boîtes n'était effective que dans 51,1\% des FOSA de l'Adamaoua et du Nord. (Tableau 1).

À l'exception de l'Hôpital Régional de Garoua, ces boîtes de sécurité étaient remplies à bord avec des débordements dans les autres hôpitaux où des bouteilles ou poubelles en plastique étaient utilisées pour le recueil des déchets piquants et tranchants (Figs. 1).

\begin{tabular}{|l|l|l|l|l|l|l|l|}
\hline Tableau I Résultats globaux / Global résultats & & & & & & \\
\hline $\begin{array}{l}\text { Résultats } \\
\text { FOSA PBF et } \\
\text { Non PBF } \\
\text { systématique }\end{array}$ & $\begin{array}{l}\text { Tri inadapté } \\
\text { syibilitécurité }\end{array}$ & $\begin{array}{l}\text { Utilisation } \\
\text { boîtes de } \\
\text { sécurité }\end{array}$ & $\begin{array}{l}\text { Existence } \\
\text { lieu stockage } \\
\text { sécurisé }\end{array}$ & $\begin{array}{l}\text { Disponibilité } \\
\text { des EPI }\end{array}$ \\
\hline $\begin{array}{l}\text { FOSA } \\
\text { Est }\end{array}$ & $17(19,8 \%)$ & $13(100 \%)$ & $11(12,8 \%)$ & $18(20,9 \%)$ & $16(18,6 \%)$ & $18(20,9 \%)$ & $13(15,2 \%)$ \\
\hline $\begin{array}{l}\text { FOSA } \\
\text { Nord-Ouest }\end{array}$ & $10(11,6 \%)$ & $12(00 \%)$ & $6(7 \%)$ & $19(22,1 \%)$ & $11(12,8 \%)$ & $15(17,4 \%)$ & $10(11,6 \%)$ \\
\hline Ad-amaoua & $6(7 \%)$ & $5(100 \%)$ & $5(5,8 \%)$ & $15(17,4 \%)$ & $8(9,3 \%)$ & $13(15,2 \%)$ & $7(8,2 \%)$ \\
\hline Nord & $4(4,6 \%)$ & $7(100 \%)$ & $5(5,8 \%)$ & $12(13,9 \%)$ & $6(7 \%)$ & $10(11,6 \%)$ & $6(7 \%)$ \\
\hline Total & $40(46,5 \%)$ & $37(100 \%)$ & $27(31,4 \%)$ & $71(82,5 \%)$ & $44(51,2 \%)$ & $61(70,9 \%)$ & $39(45,5 \%)$ \\
\hline
\end{tabular}

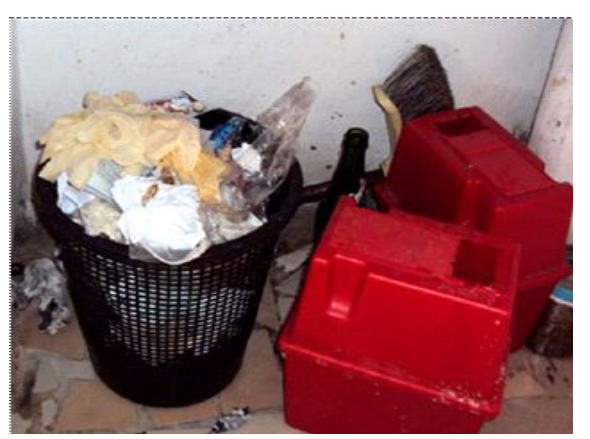

Fig. 1 Poubelle en plastique remplie de DBM non triés avec des DBM au niveau du sol à côté de boites de sécurité vides / Plastic bottles filled with unsorted biomedical waste in with biomedical waste on the floor next to empty safety bottles. 
Le système de codage par couleur n'est effectif dans aucune FOSA (usage du rouge pour les déchets anatomiques, l'orange pour les déchets d'animaux et le jaune pour les déchets de sang et fluides, les déchets piquants et tranchants et les déchets de laboratoires).

Toutefois, les déchets anatomiques étaient conditionnés dans des sacs plastiques déposés dans des poubelles plastiques munies de couvercles au niveau de tous les FOSA, sauf au niveau des CMA ou des SCI où ils étaient jetés dans les fosses à ciel ouvert. Les DBM d'activités de soins à risque infectieux étaient conditionnés dans des sacs.

La collecte des DBM était journalière au sein de tous les hôpitaux, sauf au niveau des SCI où elle est réalisée tous les mardis et vendredis avec acheminement des DBM.

Un lieu de stockage sécurisé existait dans 70,9\% ( $n=61)$ des services alors que le lieu de stockage central était non sécurisé, à ciel ouvert avec des DBM jonchant le sol au niveau de tous les hôpitaux sauf au niveau de l'Hôpital Central de Garoua (Fig. 2).

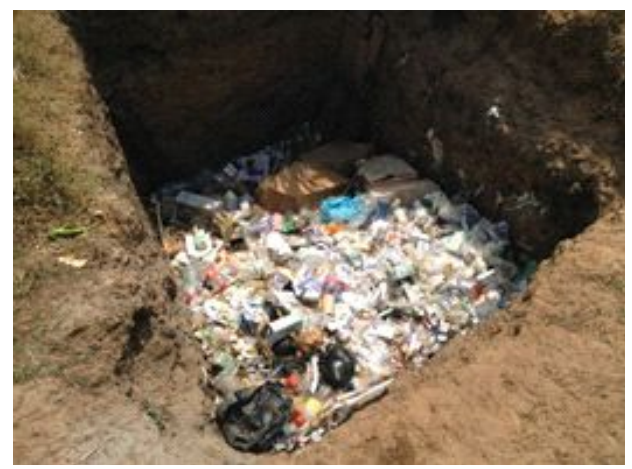

Fig. 2 Lieu de stockage central avec mélange des DBM, restes alimentaires et DAOM/Central storage place with mixing of DBM, food scraps and DAOM.

Le transport des DBM à l'intérieur des services se faisait par manutention manuelle dans 55,8 \% $(\mathrm{n}=48)$ des services, à l'aide des brouettes servant au transport des patients dans 22,1\% (n $=19$ ) alors que le transport vers le lieu de stockage central se faisait grâce aux chariots dans 67,4 \% $(\mathrm{n}=58)$ des services et par des cartons dans 33,7 $\%(\mathrm{n}=29)$.
Au niveau des cinq hôpitaux, l'évacuation des DAOM est assurée par des entreprises privées jusqu'à la décharge à ordures

Les déchets fluides étaient dilués dans l'eau de javel, contenus dans des bidons, puis déversés dans le système des égouts.

Les lames en verre étaient récupérées et réutilisées après désinfection et autoclavage.

Les déchets piquants/tranchants, anatomiques et les DASRI solides étaient incinérés au niveau des fosses à ciel ouvert, sauf au niveau de l'hôpital Central de Garoua et de Bamenda qui disposent d'incinérateur électrique. Au niveau des autres FOSA PBF, ces déchets étaient brûlés dans un four artisanal. Les incinérateurs utilisés au niveau des FOSA étaient de vieux modèles produisant beaucoup de fumées (Fig. 4).
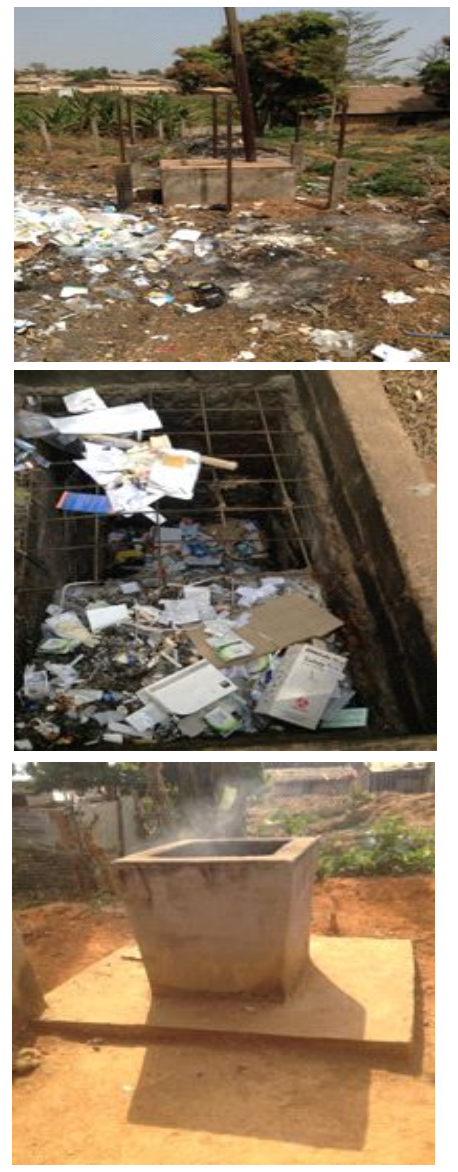

Fig4. Incinérateur et four artisanal pour l'élimination des $\mathrm{DBM} /$ Incinerators and artisanal ovens for biomedical waste 
Les conditions de travail étaient jugées mauvaises par $81,3 \%(\mathrm{n}=61)$ des travailleurs interrogés et bonnes par $18,7 \%(\mathrm{n}=14)$.

Les équipements de protection individuelle (EPI) étaient disponibles dans 45,5\% $(\mathrm{n}=39)$ des FOSA PBF avec des gants de protection en caoutchouc dans tous ces services, des masques dans $74,3 \%(\mathrm{n}=29)$ d'entre eux, des tabliers dans $25,6 \%(\mathrm{n}=10)$ et des bottes dans $15,4 \%$ $(\mathrm{n}=6)$.

Par contre dans les FOSA non PBF les EPI, des gants de protection en caoutchouc, des masques, des tabliers dans et des bottes étaient tous absent. Les connaissances sur la gestion des DBM étaient jugées insuffisantes par 62,6\% $(\mathrm{n}=47)$ des personnes interrogées et les risques sanitaires liés aux DBM connus par $80 \%(\mathrm{n}=60)$ d'entre eux dans tous les FOSA.

Au niveau de la formation sur la gestion des DBM, une séance a été organisée pour chaque catégorie professionnelle des FOSA.

\section{Discussion}

La faible proportion de déchets anatomiques notée dans notre enquête n'est pas retrouvée dans une étude antérieure similaire où ces types de déchets étaient les plus importants. Il faut également signaler que, chez les musulmans, les déchets anatomiques comme les membres ou segments de membres sont souvent récupérés par les familles pour être enterrés dans un cimetière [3].

Le tri des DBM est inadapté dans la majorité de formations sanitaires enquêtées (entre $75 \%$ dans les FOSA Non PBF et 58,3 \% dans les FOSA PBF), sauf à l'Hôpital Central de Garoua où seuls 5,5\% des services sont concernés [9].

Au niveau des FOSA PBF et non PBF, le comité d'hygiène est géré théoriquement par l'Établissement.

L'absence d'une utilisation du système de codage dans $100 \%$ des formations hospitaliers obéit aux mêmes explications fournies dans le domaine du tri. Ce codage, en permettant l'identification et la séparation des DBM, réduit de façon significative la quantité de déchets nécessitant un traitement spécial et le coût de ce traitement [7]

Une étude en 2008 par MINSANTE propose un système de tri en trois catégories avec une séparation des déchets domestiques, des déchets piquants et tranchants, et des déchets infectieux. Malgré leur disponibilité dans 82,5\% des FOSA Visités, les boîtes de sécurité ne sont utilisées que dans la moitié d'entre eux $(51,1 \%)$. Cette utilisation moyenne, l'observation de boîtes remplies à bord avec des débordements et l'utilisation de bouteilles ou de poubelles en plastique pour le recueil soulignent l'absence ou le non-respect des consignes par le personnel et surtout l'existence de ruptures dans l'approvisionnement de ce matériel [12].

Le transport des DBM à l'intérieur des services et vers le lieu de stockage central constitue une étape à risque car effectué par manutention manuelle ou à l'aide de cartons et brouette. En effet, ce transport devrait suivre un circuit éloigné des zones fréquentées par les patients et visiteurs et s'effectuer avec le maximum de sécurité à l'aide de chariots sanitaires ajustables. Ce type de chariot doit être facile à charger, à décharger, à nettoyer et être fabriqué à moindre coût par l'artisanat local [5].

Une étude sur la gestion des DBM à l'hôpital Jamot et au District de Santé de Biyem Assi dans la région du Centre au Cameroun a montré également des conditions de transport à risque avec l'utilisation des tables roulantes et des poubelles portées sur le dos ou la tête.

La situation désastreuse notée dans la collecte et le transport des DBM engendre des risques professionnels (accident de travail, maladie professionnelle, usure prématurée au travail) et des risques infectieux pour les patients, visiteurs et animaux [7].

Le stockage des déchets à risque exige des conditions spéciales, à savoir des locaux sécurisés, 
fermés à clé, faciles à nettoyer, bien éclairés, ventilés et interdits d'accès à toute personne étrangère au service.

$\mathrm{Si}$, dans notre étude, des lieux de stockage n'existent pas dans toutes les structures, avec cependant une sécurisation notée, il a même aussi noté dans l'étude de Daoudi menée au niveau de l'hôpital Hassan-II d'Agadir et Bidias Jacob où on note une absence totale de lieux de stockage [1-8].

L'incinération par de vieux modèles d'incinérateurs constitue le mode de traitement des déchets à risque le plus utilisé dans nos structures, sauf au niveau de l'hôpital Central de Garoua où on pratique le brûlage dans un incinérateur électrique. Lors des activités d'incinération, se dégagent des émanations chargées de métaux lourds, gaz nocifs et particules organochlorées qui polluent l'air et engendrent des risques de dégradation de l'environnement, contamination des eaux et sols et intoxication des populations et animaux [10-15].Les EPI sont disponibles dans 45,3\% des services seulement. Selon l'OMS, plus de 100000 cas d'infections liées aux procédures de soins sont observés chaque année en Angleterre alors que, dans les pays en développement, ces cas sont majorés de 2 à 20. De même, la manipulation des déchets sanitaires infectés par le VIH est responsable de $0,2 \%$ des cas de transmission au niveau mondial selon des statistiques datées de 2010[12].

Les conditions de travail, jugées mauvaises par $81,3 \%$ des travailleurs interrogés, engendrent un stress, facteur d'aggravation des risques liés aux DBM, et un climat d'insécurité source de conflits sociaux [16-19].

Le faible niveau des connaissances sur la gestion des DBM, noté chez 62,6 \% des FOSA enquêtés, cela pourra nécessiter la mise en application du Comité d'Hygiène Hospitalier $(\mathrm{CHH})$ et des formations dans la gestion des DBM dans les formations sanitaires. Cette formation doit être adaptée à la réalité vécue au niveau de chaque hôpital, mais surtout basée sur la correction des comportements à risque et l'utilisation judicieuse du matériel existant. De même, une sensibilisation doit être menée en direction des responsables des hôpitaux pour une application effective de la législation et la création de services de médecine du travail, structures essentielles dans la connaissance et la prévention des risques professionnels[8-14].

\section{Conclusion}

Au Cameroun, il n'existe pas une législation sur la gestion des DBM mais sur la gestion des déchets en général qui stipule dans son décret N²012/ 2809/PM du 26 septembre 2012 relatif aux conditions de tri, de collecte, de stockage, de transport, de récupération, de recyclage, de traitement et d'élimination finale des déchets qui stipule à son article 12 (1) que: les déchets médicaux et pharmaceutiques font l'objet d'une gestion spécifique visant à éviter toute atteinte à la santé de l'homme.

Cependant, force est de reconnaitre que cette législation n'est pas appliquée, car méconnue des responsables et professionnels de la santé. À la lumière de cette étude, nous recommandons :

- le renforcement du cadre législatif et réglementaire ;

- la mise en œuvre du système de stockage, de traitement et de gestion des déchets à risque ;

- la réduction des déchets à la source et la maîtrise de procédé de traitement ;

- la mise en place d'un système de suivi et de contrôle pour assurer la conformité

La gestion défectueuse des DBM est une réalité au niveau des structures hospitalières au Cameroun où des dysfonctionnements sont notés à toutes les étapes. Cette situation entraîne des risques pour la santé et la sécurité du personnel de la santé, des patients, des populations et une dégradation de l'environnement. 
La réponse à cette gestion défectueuse passe par l'application effective de la législation sur la gestion des DBM, la médecine du travail, l'adoption de programmes de gestion des DBM adaptés et réalisables et la formation du personnel.

\section{Références}

1. Anonyme, 2000. OMS, "Les déchets liés aux soins de santé", aide-mémoire 2 n53; 2000.

2. Anonyme, 2002.Stratégie Nationale sur la sécurité des Injections et la Gestion du Matériel d'Injection utilisé, MINSANTE, République du Cameroun. Pp30-33

3. Anonyme, 2004. Description de la situation des déchets sanitaires au Cameroun, Analyse de la situation actuelle effectuée lors d'une mission, EPOS Health Consultants/ KFW.pp 12-15

4. Anonyme, 2005a. Plan national de gestion des soins médicaux «manuel d'aide à la décision »P.N.U.E et OMS, pp 3-36.

5. Anonyme, 2007.Rapport DPS/ MINSANTE. Inventaire des déchets hospitaliers au Cameroun.P.11

6. Anonyme, 2007. Stratégie Nationale de Gestion des Déchets (SNGD), 2007, MINEP, République du Cameroun. P25

7. Bidias J., 2013. Evaluation de la gestion des déchets solides de l'hôpital Jamot de Yaoundé et de l'hôpital de district de Biyem Assi : Impacts environnementaux et sociaux

8. Daoudi, 2008. Evaluation de la gestion des déchets solides médicaux et pharmaceutiques à hôpital Hassan II d'Agadir, pp10-25.

9. Faye P (1997) Évaluation du projet de collecte, évacuation et traitement des ordures ménagères. Mémoire de génie sanitaire. CES de santé publique, Institut de santé et développement (ISED), UCAD Sénégal, $41 \mathrm{p}$

10. Gayathri VP, Pokhrel K (2005) biomedical solid waste management in an Indian hospital: a case study. Waste Manag 25(6):592-9
11. Kane MS (2005) Inventaire des sources de dioxines en Afrique subsaharienne. Identification des déchets précurseurs de dioxines en fonction des pratiques de gestion endogènes (Sénégal). Centre régional de la convention de Bâle pour les pays africains francophones, Rapport, 14 $\mathrm{p}$

12. Manga Blaise, 2009. Etude sur les systèmes appropriés de traitement des déchets médicaux dans le cadre du programme national de développement participatif, pp 6-16.

13. Maton P. "Le raccordement des rejets hospitaliers au système d'assainissement collectif', Techniques hospitalières, Mars 1999, n634, pp 62-63.

14. Mbog Mbog S,2013. Evaluation de la gestion des déchets liquides hospitalier dans le Centre Hospitalier Universitaire de Yaoundé (CHU) : Cas des Eaux Usées

15. Mounier M. et DENIS F. "Risques épidémiologiques liés aux déchets d'activités de soins", Techniques hospitalières, Décembre 1998, $n^{\circ} 632$,

16. Mbengue MF (1999) Déchets biomédicaux en Afrique de l'Ouest : problèmes de gestion et esquisse de solution, IAGU- PGU, pp 13-27

17. Ndiaye P, Fall C, Diedhiou A, et al (2003) Gestion des déchets biomédicaux (DBM) au centre hospitalier régional (CHR) de Ziguinchor. Cahiers d'études et de recherches francophones/ Santé 13(3): 171-6

18. Prüss A, Giroult E, Rushbrook P (1999) Safe management of wastes from health-care activities. WHO, Geneva, 230 p

19. Sanogo M, Sokona F, Guindo S, et al (2007) Contribution à la mise en place d'un système de gestion durable des déchets biomédicaux à l'hôpital Gabriel-Touré (Mali). Pharm Hosp 42:143-7 